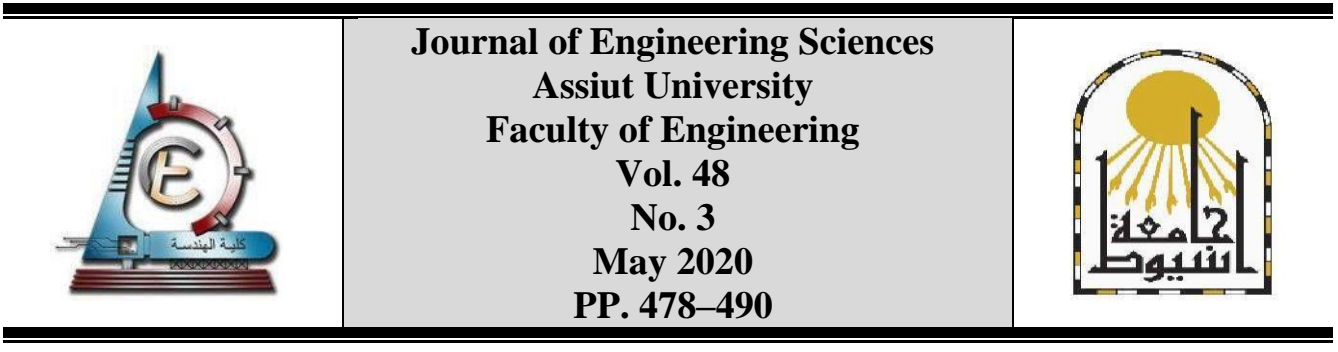

تأثير التوجيه واختلاف الأدوار على الأداء الصراري للفراغات الداخلية

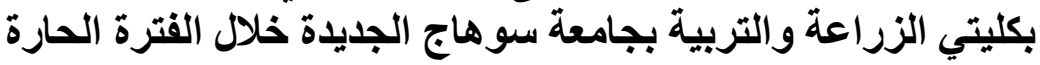

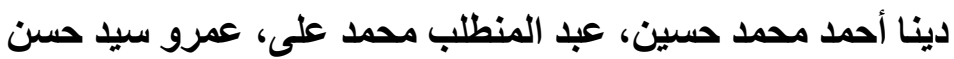

قسم الهندسة المعدارية _كلبة الهنسة - جامعة أسبوط

Received 23 December 2019; Accepted 16 January 2020

الملخص

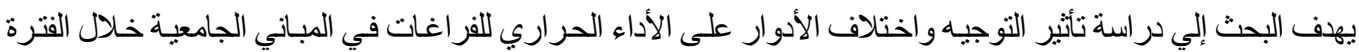

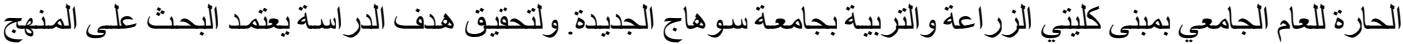

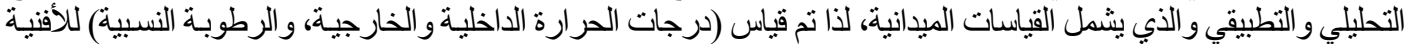

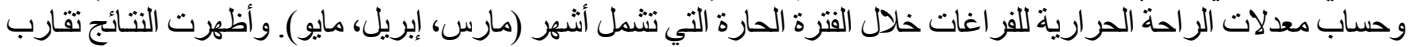

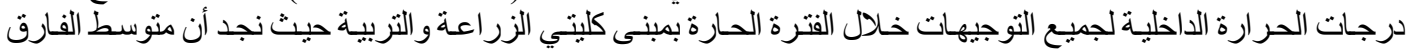

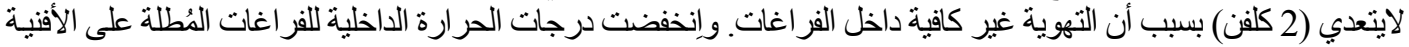

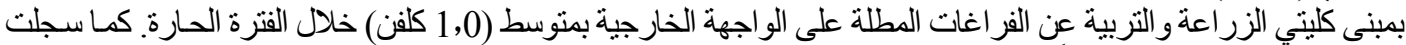

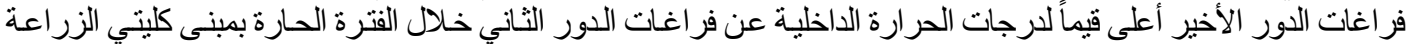

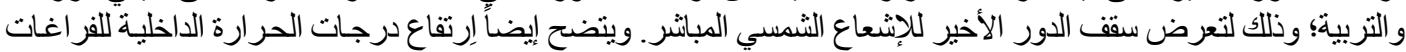

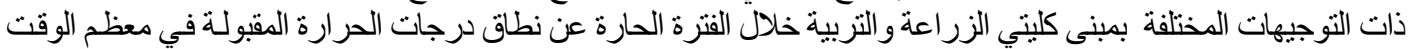

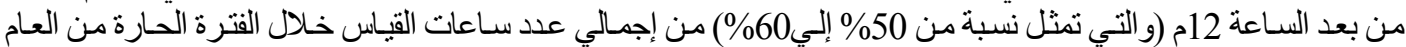

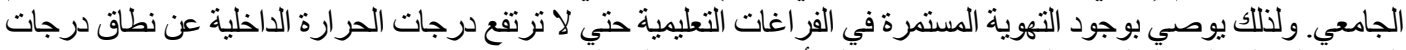
الحرارة الداخلية المقبولَّة لراحة الطلاب مما يؤثر علي أداءهم وتحصيلهم الدراسي.

الكلمات المفتحية: الراحة الحراريةـ المباني الجامعيةـ الأداء الحراري- الفراغات التعليميةـ التوجيهـ اختلاف الأدوارـ المطل.

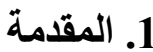

وفقًا لتغير المناخ العالمي، نحتاج لمبانٍ مُصممة بشكل جيد حتى نتمكن من تحقيق مستويات عاليـة لكفاءة المقاء

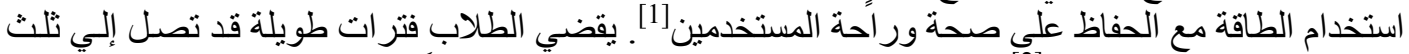

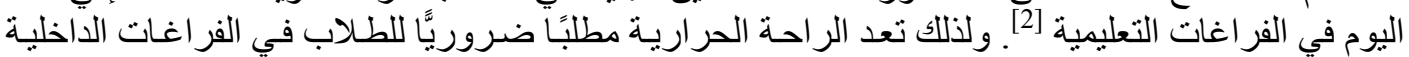

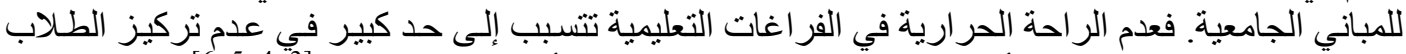

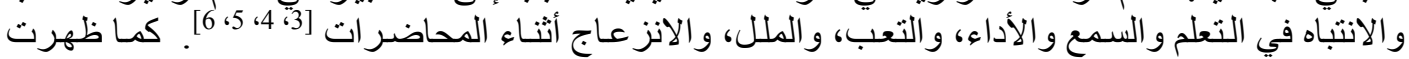

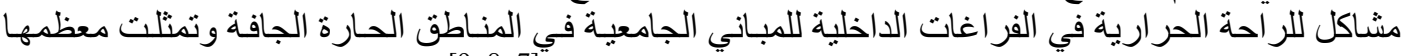

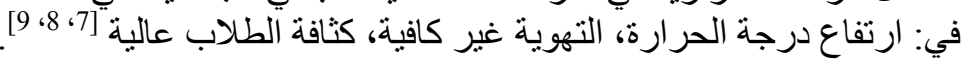

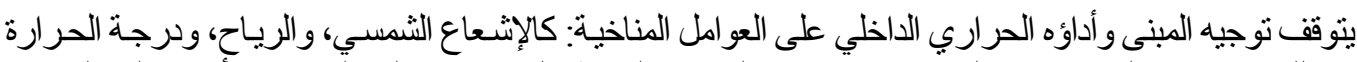

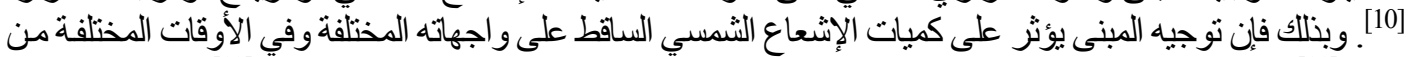

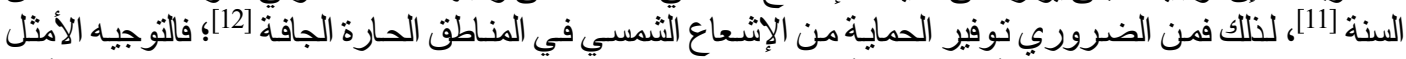

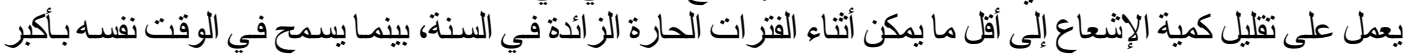




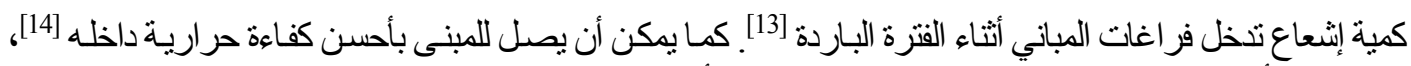

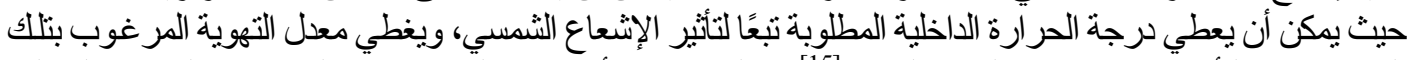

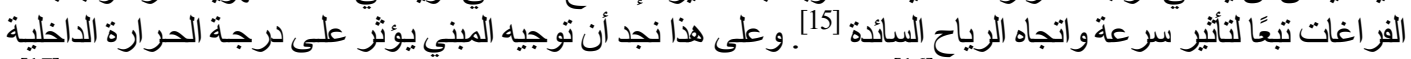

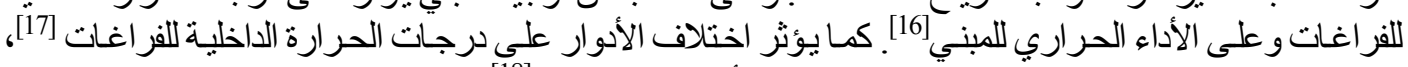

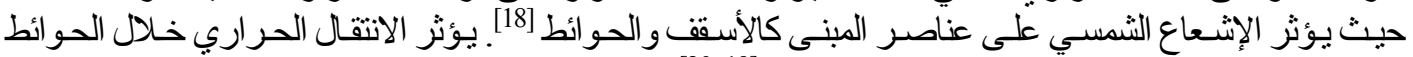

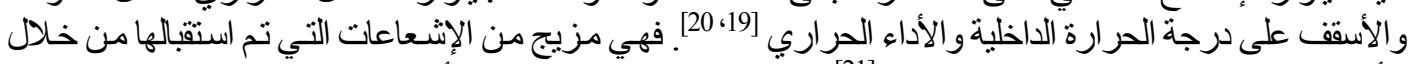

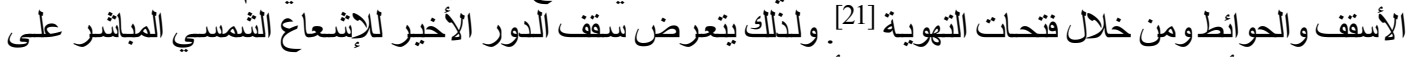

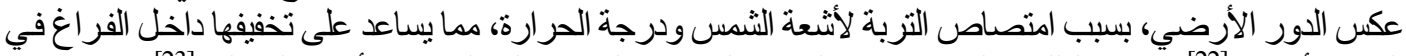
الدور الأرضي [22]. ونتيجة للتلك هناك اختلافات ملحوظة لدرجات الحرارة الداخلية بين الأدوار المختلفة [23]. ولللك أجرت بعض الدر اسـات تقييم الأداء الحراري للبيئة الداخليـة للمباني الجامعيـة [24، 25، 26، 27، 28]،

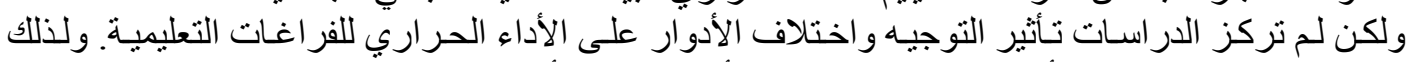

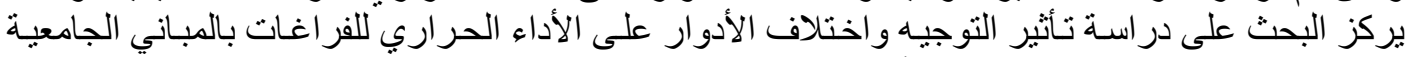

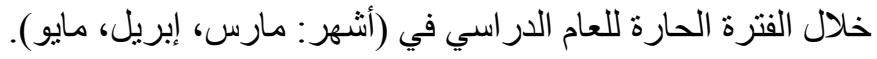
1.1 إثكالبة الدراسة

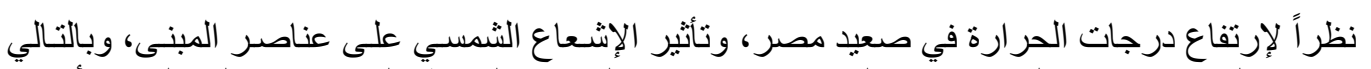

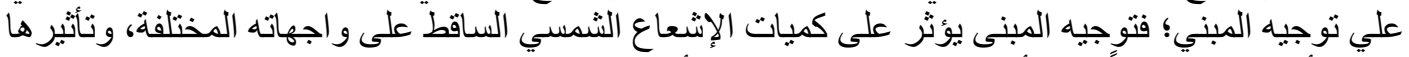

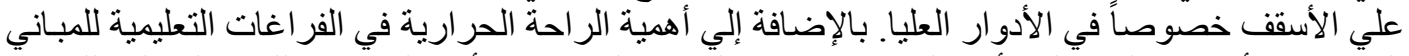

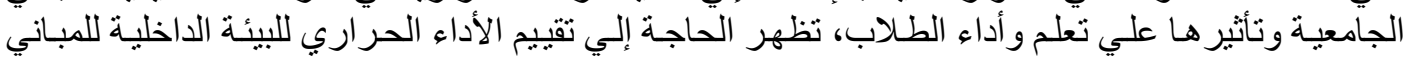

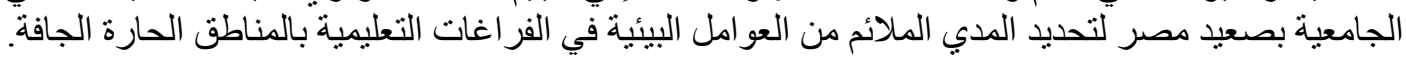

$$
\text { 2.1 2.1 العزف من الدراسة بهنة }
$$

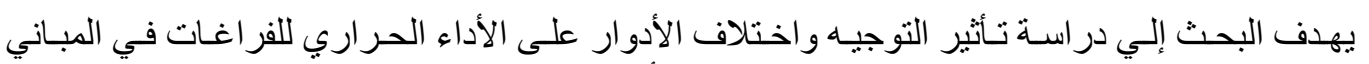

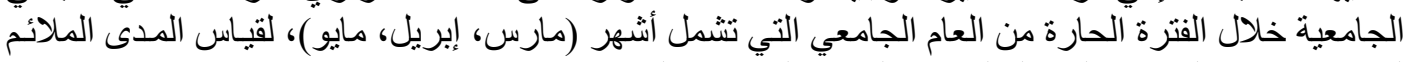

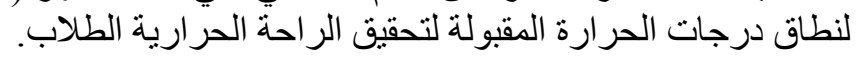

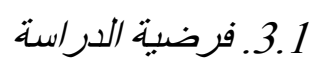

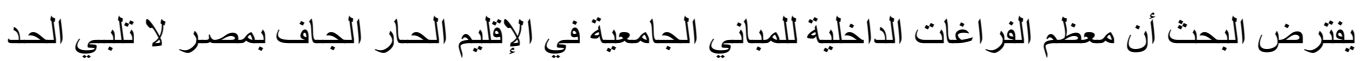
الأدني من الر احة الحر ارية لمستخدميها خلال الفترة الحارة.

$$
\text { 4.1 } 4.1
$$

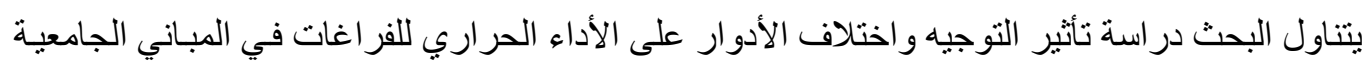

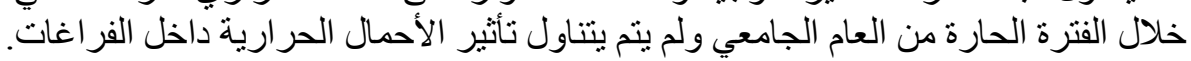

\section{تم استخدام "المنهج التحليلي " في:

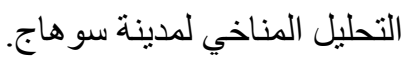

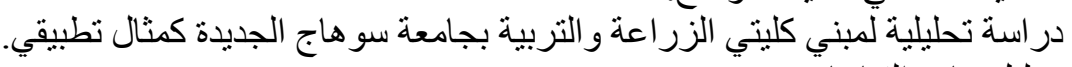
تحليل نتائج القياسات.

\section{تم استخدام "المنهج التطبيقي" في:}

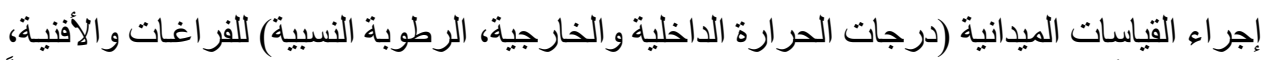

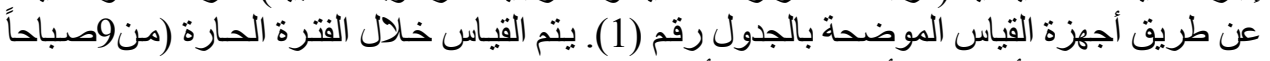
إلي 3مساءً) لمدة 6أيام خلال أثنهر (مارس، أبريل، مايو)، للعام الدراسي 2017مة 2018م. 
جول 1: الأجهزة المستخدمة في فترة القياس.

\begin{tabular}{|c|c|c|c|c|}
\hline المدي & ألإقَة & صنورةً الجهاز & الجهاز المُسئخذم & الققبأساتَ \\
\hline 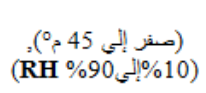 & ${ }^{0}, 1 \pm, \% 1 \pm$ & if & TR72Ui & 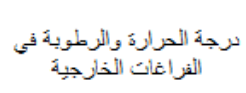 \\
\hline 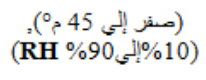 & 0 & & TR-76Ui & سرجة الحراردّ والزرطوية في \\
\hline (50-50 إلى 800 مم) & ${ }^{0} 2 \pm, \% 2 \pm$ & & $\begin{array}{l}\text { Infrared Thermometer } \\
\qquad 42515\end{array}$ & 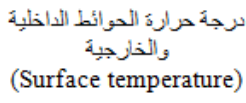 \\
\hline
\end{tabular}

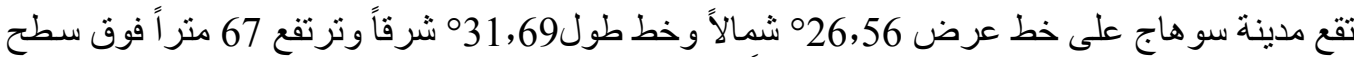

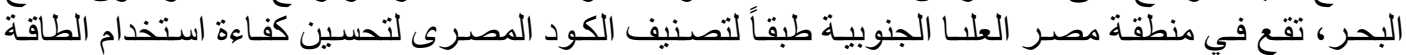

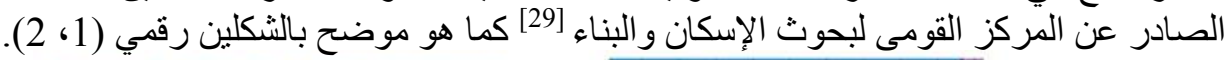

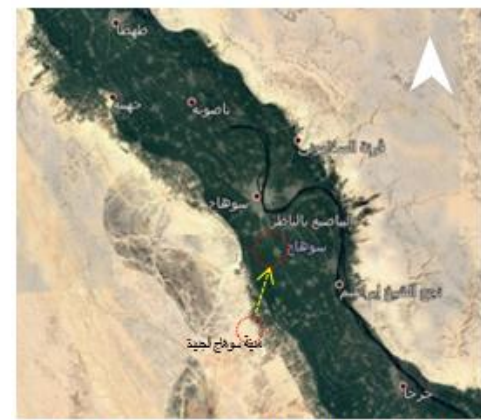

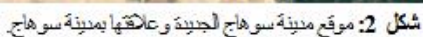

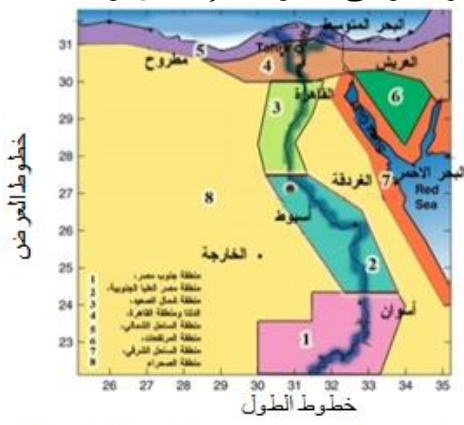

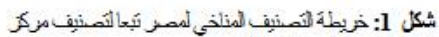
بحوت الإنسكان وليناء، 2006 [29]

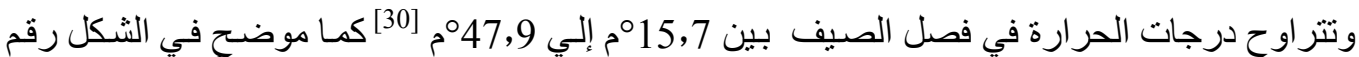

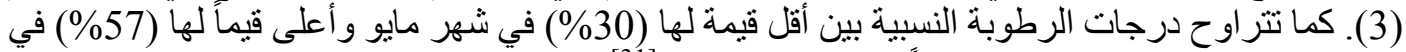

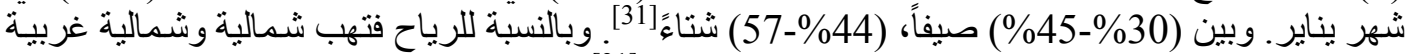

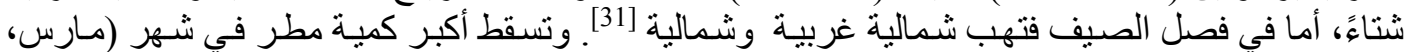

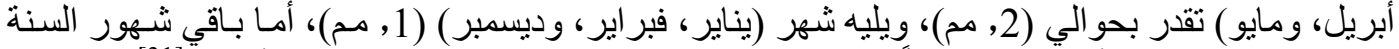

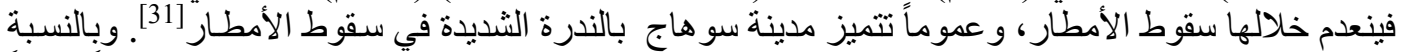

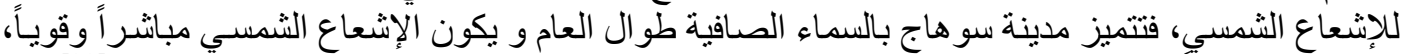

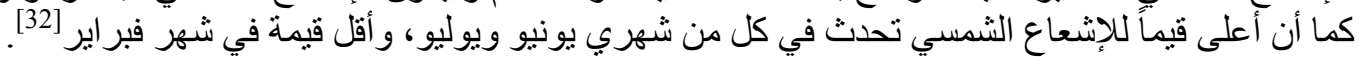

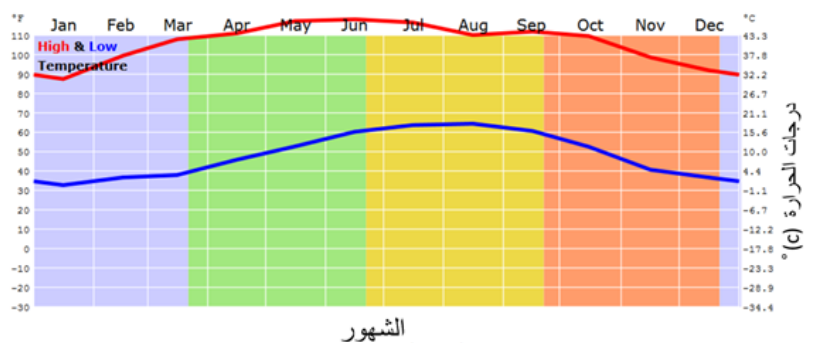

شكل 3: منوسطات القيم الشهرية لأعلى و أدنى درجة حر ارة خلال العام لمدينة سو هاج][30]. 
2.2. دراسة تحلبلية لمبني "كلية الزراعة بجامعة سوهاج الجدبية" كمثال تطبيقي تم اختيار علد من المباني مُكنملة في الاستخدامات الداخلية، وتم استبعاد عدد من الكليات لعلم تتشغيلها بكامل قوتها، ولتلك تم

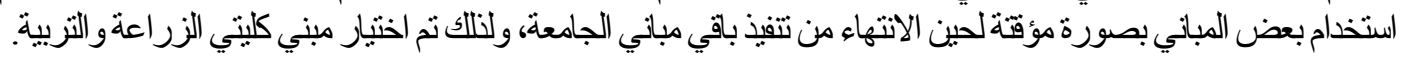
يقع مبني كلية التزبية بجوار المخل الرئيسي لجامعة سو هاج الجيبة في مدينة سوهاج الجيلة كما هو موضـح بالثنكل رقم

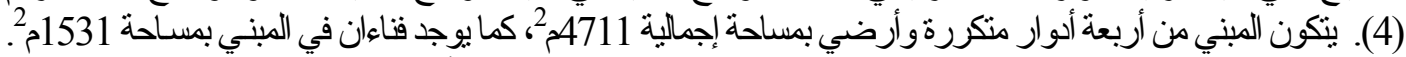

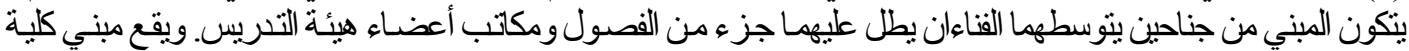

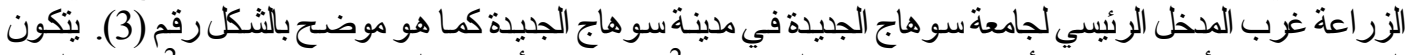

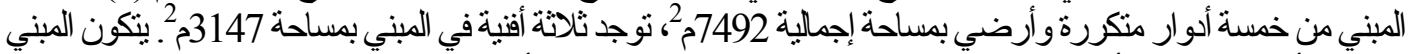
من ثثلاثة أجنحة تنوسطها أفنية يطل عليها جز هُ من الفصول و المعامل و مكاتب أعضاء هيئة التنريس.

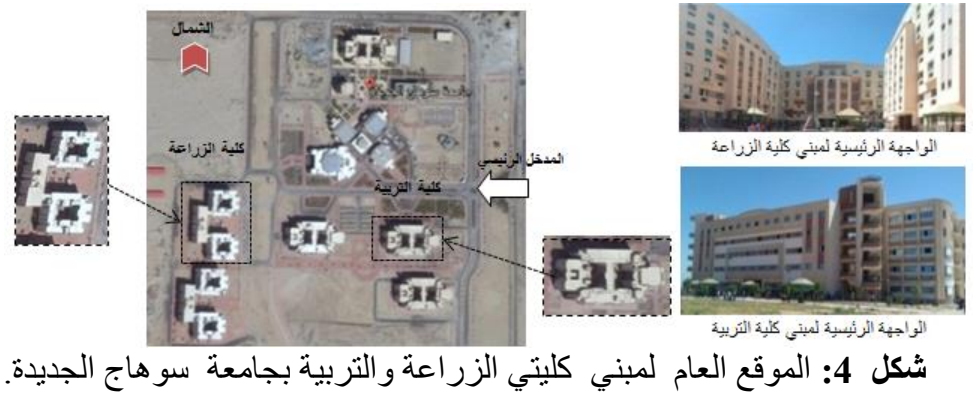

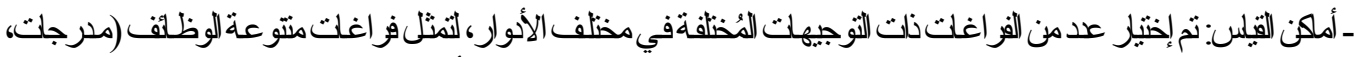

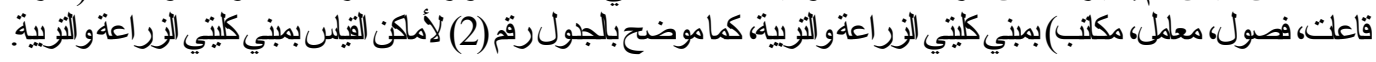
جدول 2: أماكن القياس بمبني كليتي الزر اعة و التربية.

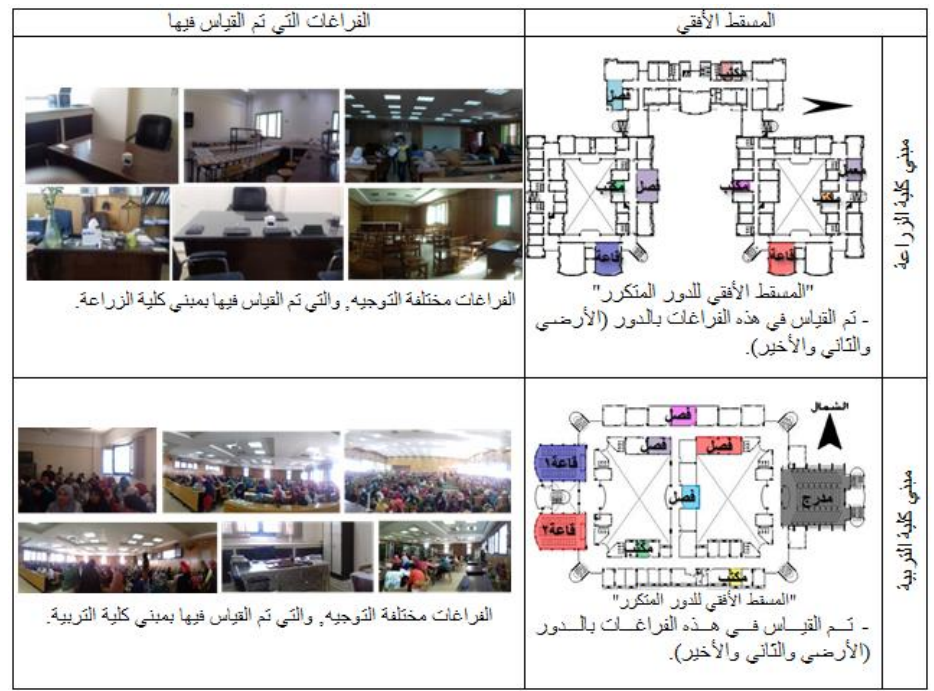

3.

1.3 ـ مبنسي كلبة الزراعة

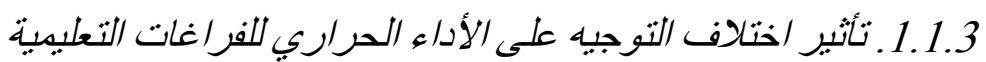

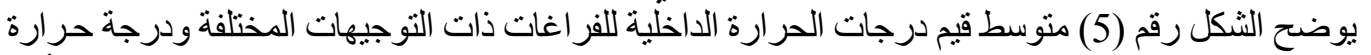

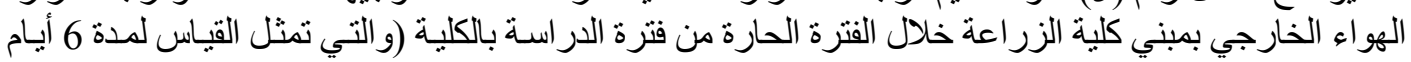




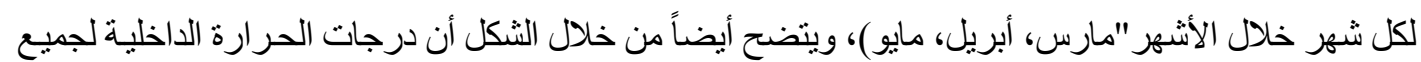

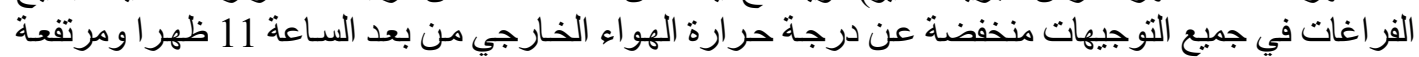

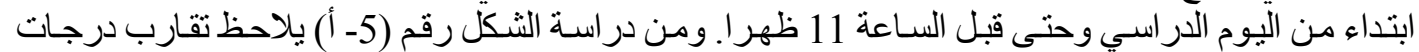

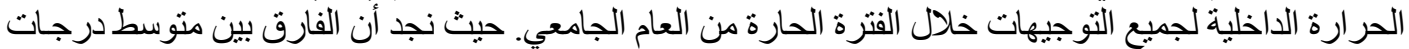

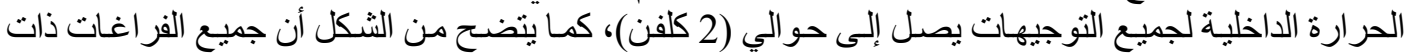

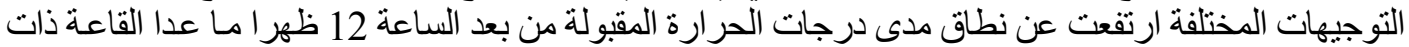

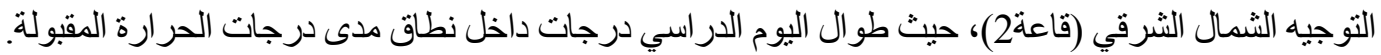

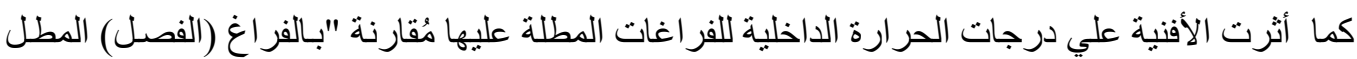

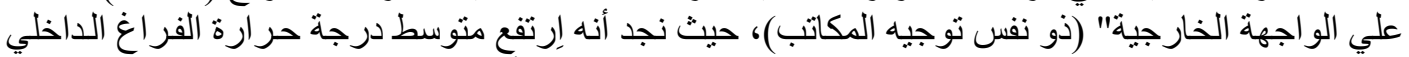

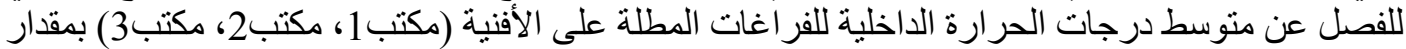

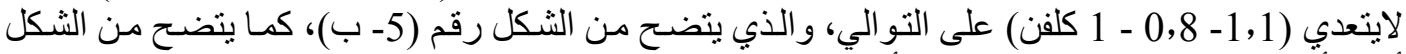

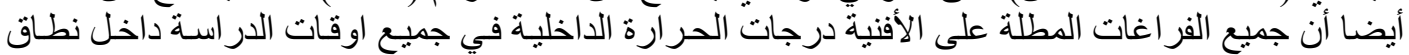

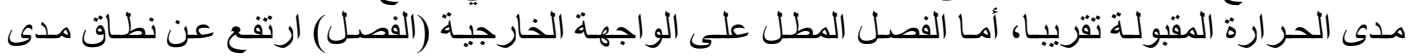
درجات الحرارة المقبولة من بعد الساعة 11 ظهر الـال.
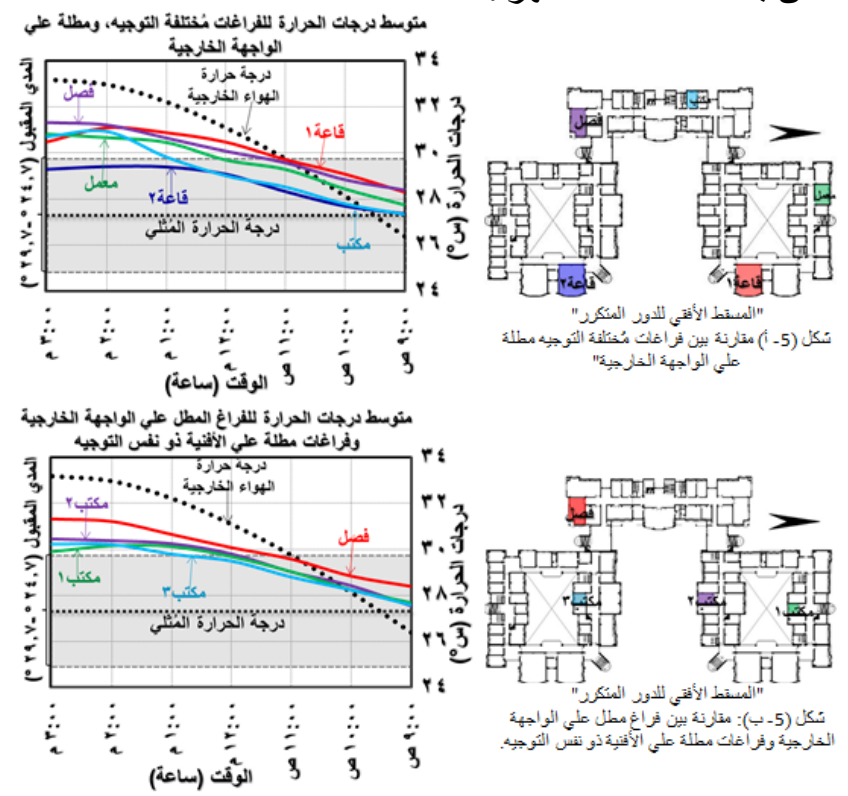

شكل 5: متوسط قيم درجات الحرارة الداخلية للفر اغات ذات التوجيهات المختلفة بمبني كلية الزر اعة في فترة القياس (من 9صل إلي 3م) خلال الفترة الحارة.

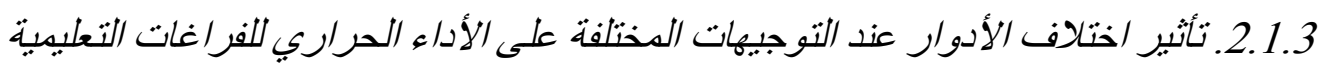
ـ ـ مقارنة بين درجات الحرارة للقاعات ذات التوات التيه الجنوب الثرقي و الثمال الشرقي:

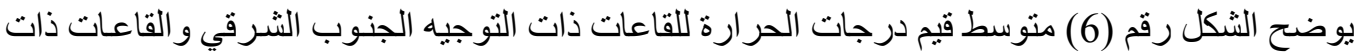

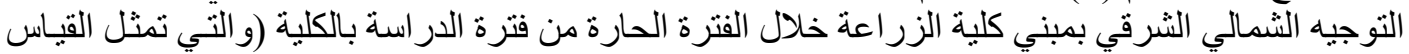

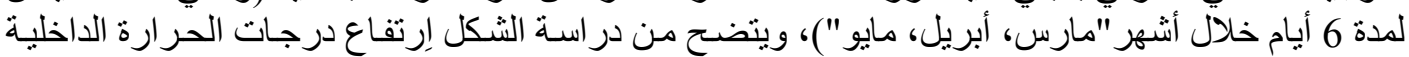

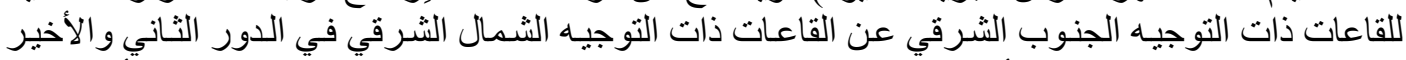

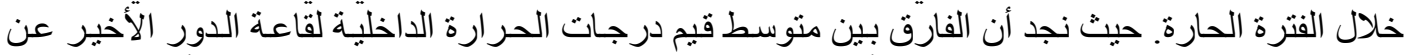

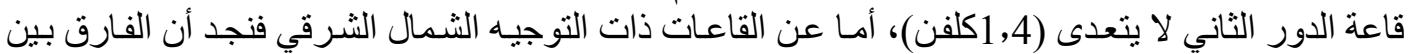

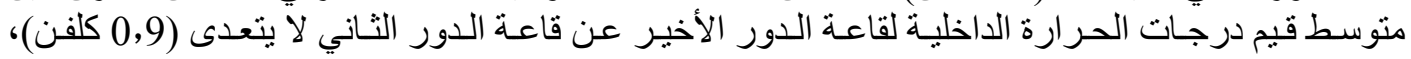


لتعرض سقف الدور الأخير للإشعاع الثمسي المباشر ـ كما يلاحظ ارتفاع درجات الحر ارة الداخلية للقاعـات

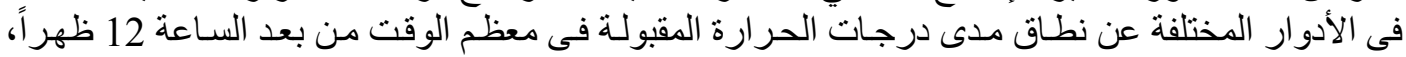

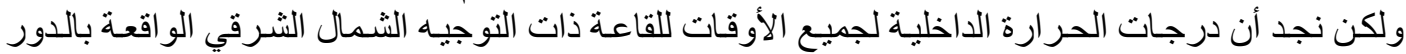

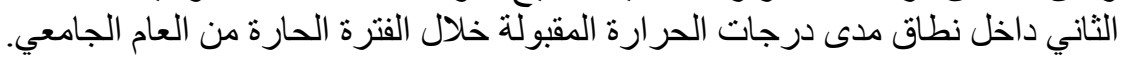
ويتضح أيضاً من خلال الثكل أن درجات الحرارة الداخلية للقاعات فى الأدوار المختلفة و التوجيهات المختلفة التهات

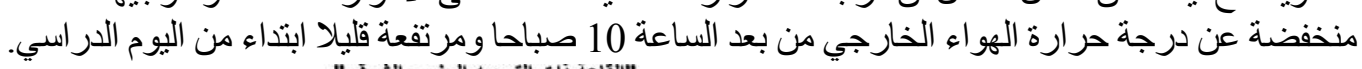
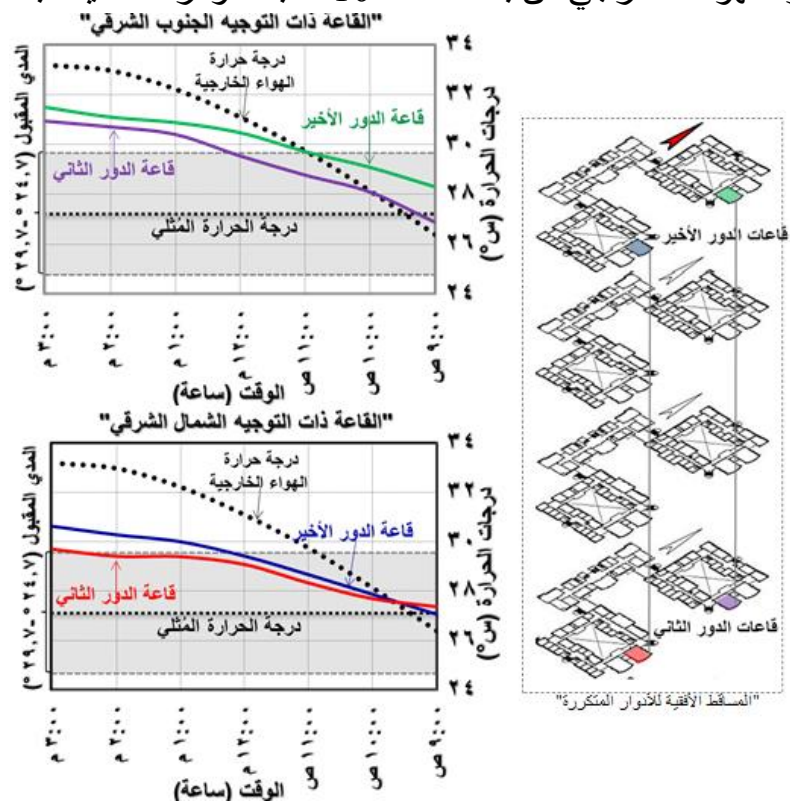

شكل 6: المساقط الأفقية للقاعات ذات التوجيه الجنوب الثـرقي و القاعات ذات التوجيه الثنمالي الثـرقي بمبني كلية الزر اعة، ومتوسط قيم درجات الحرارة لهم في الدور الثاني و الأخير خلال فترة القياس (من 9ص إلى الي 3م) في اللقترة الَحارة. ـ مقارنة بين درجات الحرارة للفر اغ المطل علي الواجهة الخارجية والمُطل على الفناء:

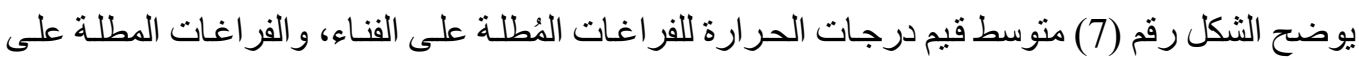

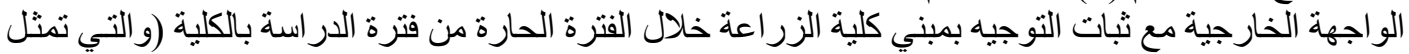

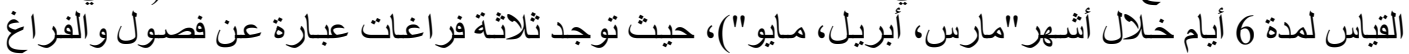

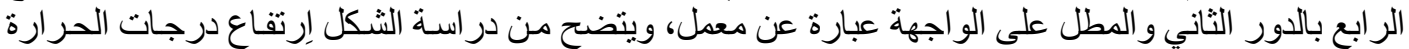

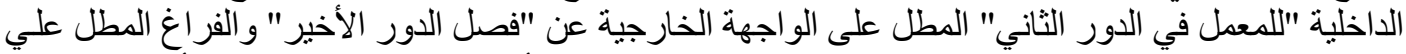

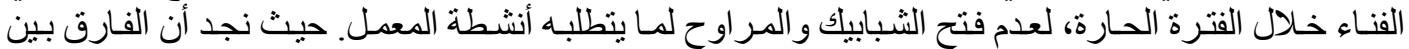

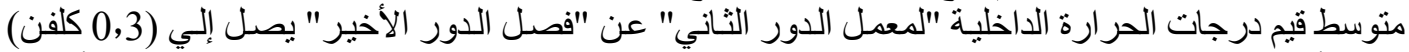

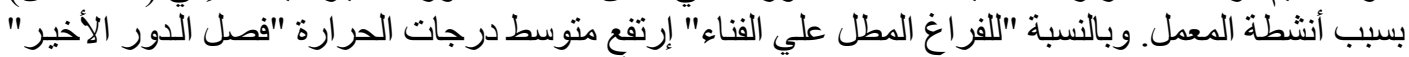

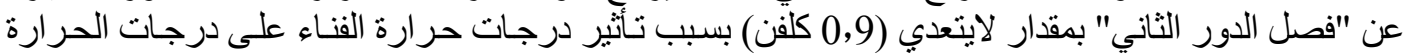

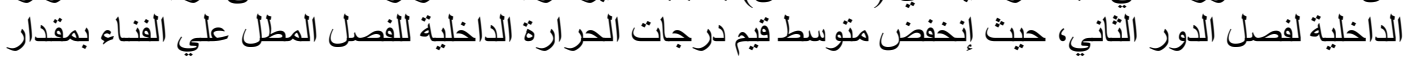
(0,7 كلفن) عن المعمل المطل علي الو اجهة الخارجية في الدور الثناني.

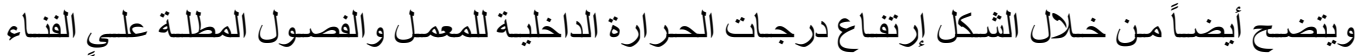

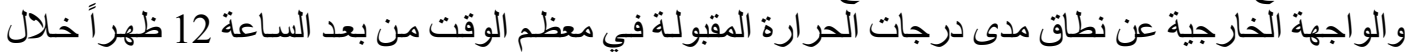

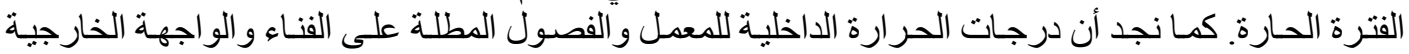
منخفضة عن درجة حرارة الهو اء الخارجي من بعد الساعة 10 صباحا ومرتفعة تقريبا ابتداء من اليوم الدراسي. 

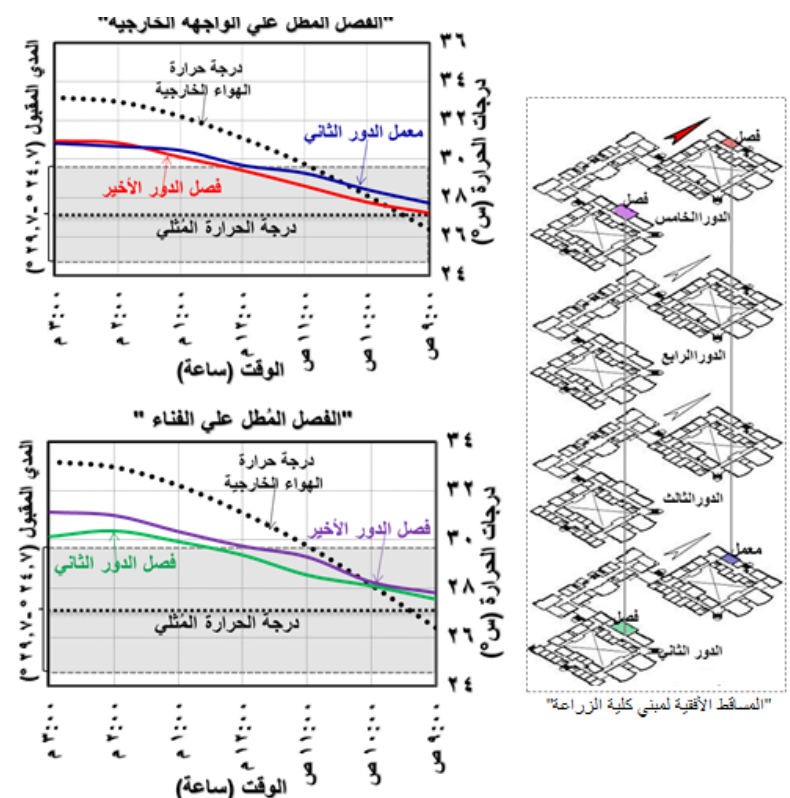

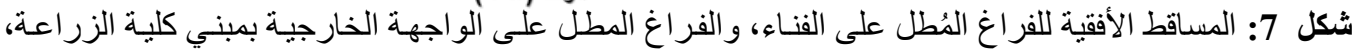

ومتوسط قيم درجات الحرارة لهما في الدور الثاني والأخير خلال فترة القياس (من وص إلى الي 3م) في الفترة الحارة.

2.3. مبني كلبة التربية

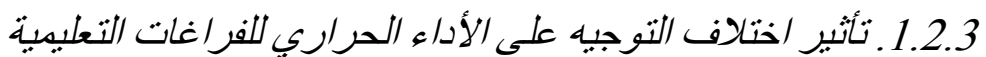

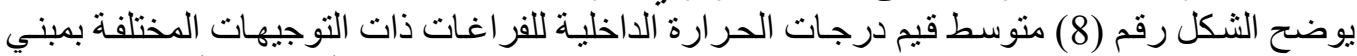

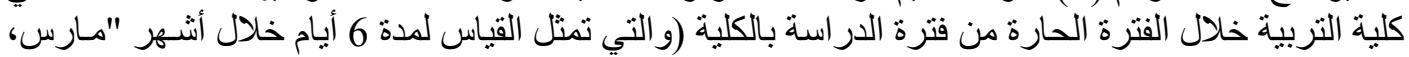

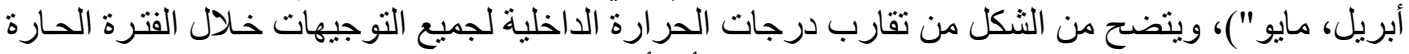

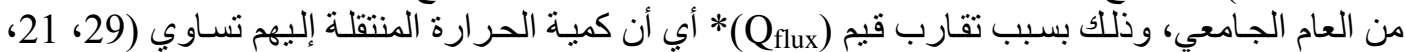

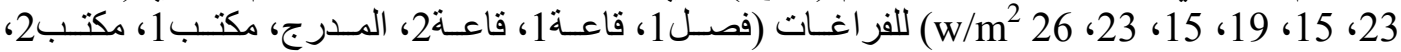

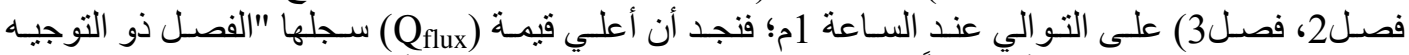

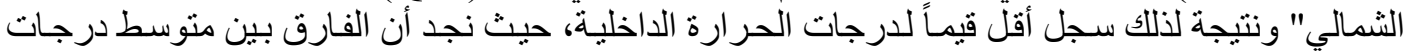
الحرارة الداخلية لجميع التوجيهات يصل إلى حوالي (1 كلفن) كما موضح بالثكل رقم (8- أ).

كما أثر الفناء علي درجات الحرارة الداخلية للفر اغات المطلة عليه مقارنة "بالفر اغ (المكتب) المطل علي

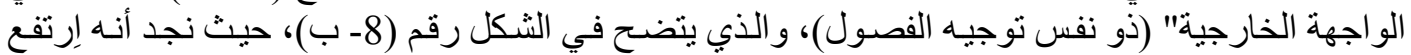

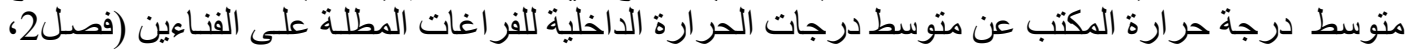

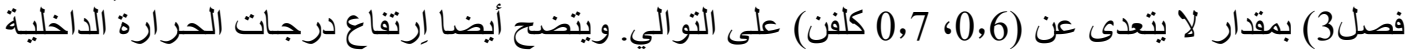

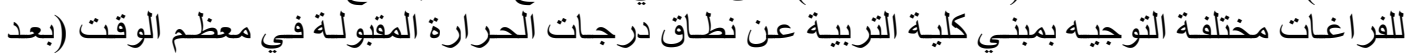
الساعة11ص) خلال الفترة الحارة من العام الجامعي. 

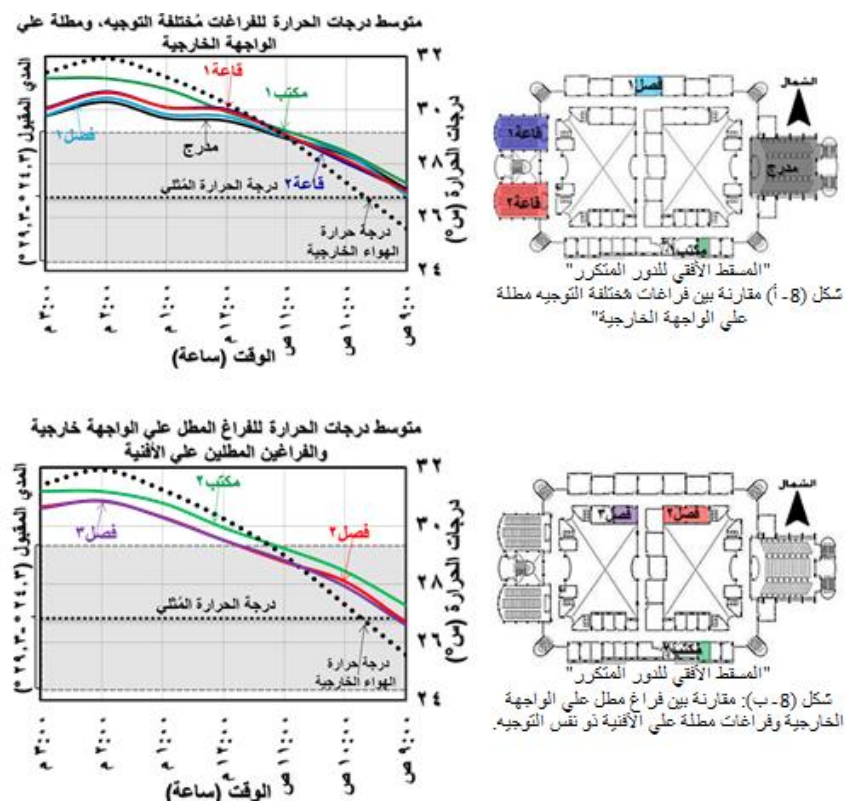

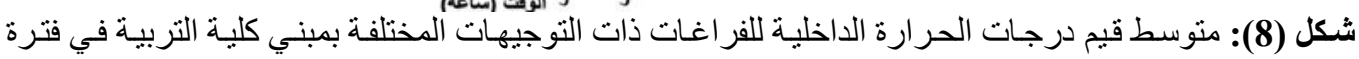
القياس (منوص (8): مثن 3م) خلال الفترة الحارة.

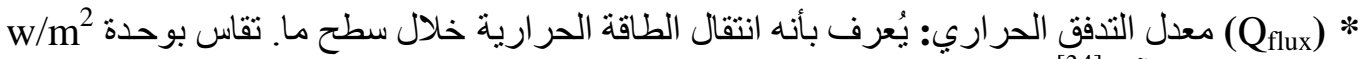
[33]، ويتم حسابه كالآتي [34] معدل:

$$
\mathrm{Q}_{\text {flux }}=\mathrm{K}^{*} \Delta \mathrm{T} / \mathrm{x}
$$

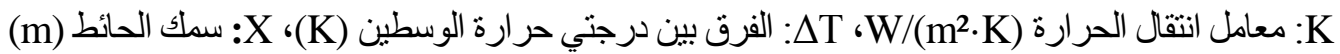

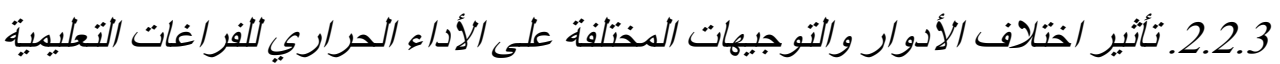

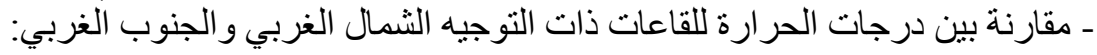

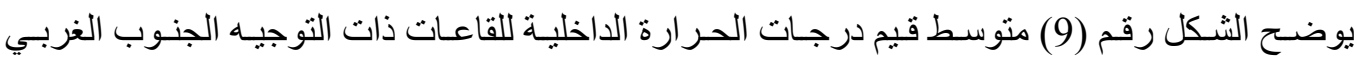

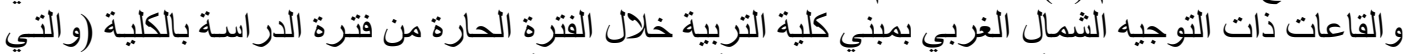

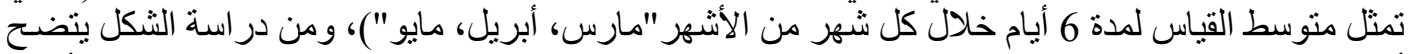

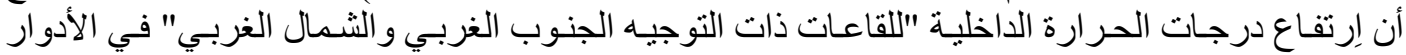

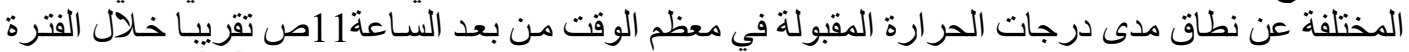

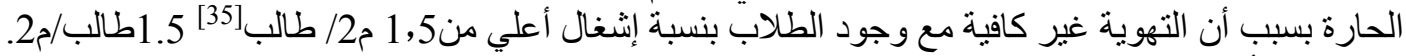

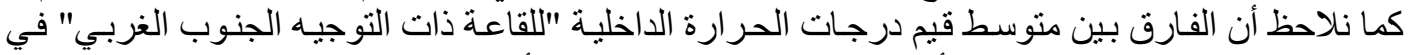

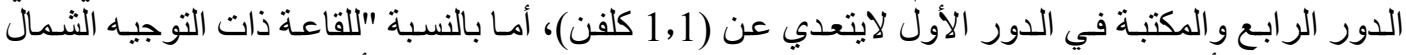

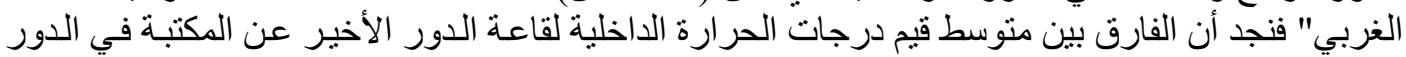
الأول لايتعدي عن (0,7 كلفن). 

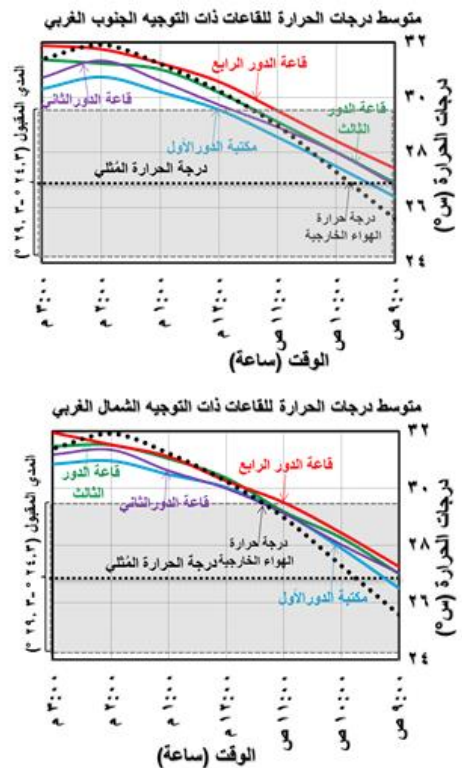

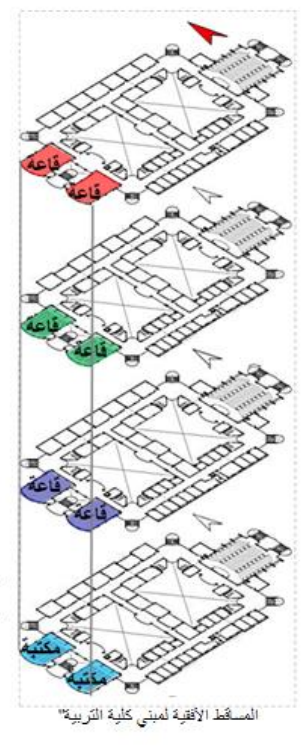

شكل 9: المساقط الأفقية للقاعات ذات التوجيه الثمال الغربي والقاعات ذات التوجيه الجنوب الغربي بمبني كلية

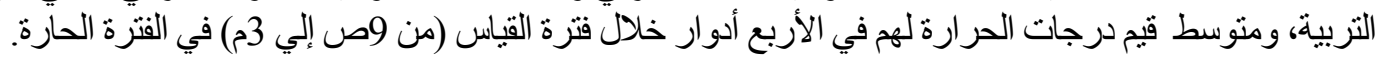
ـ مقارنة بين درجات الحرارة للفر اغ المطل علي الواجهة الخارجية و المُطل على الفناء:

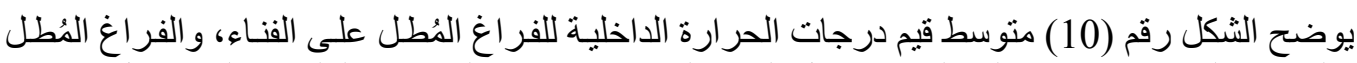

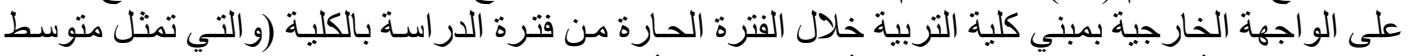

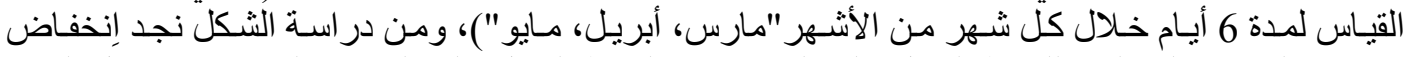

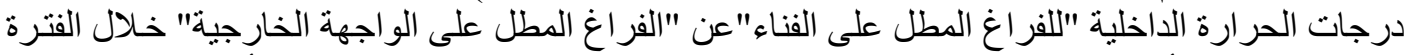

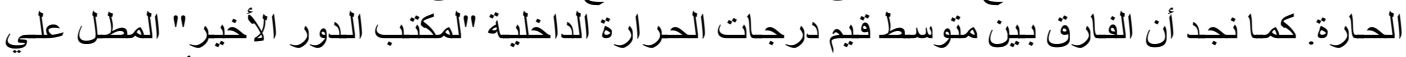

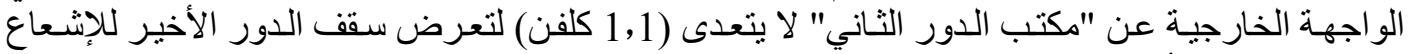

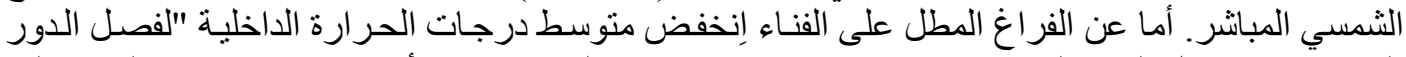

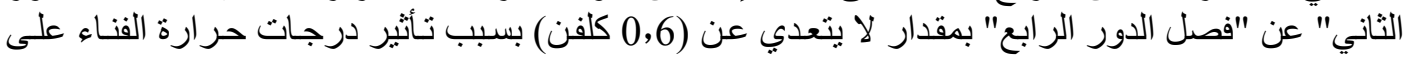

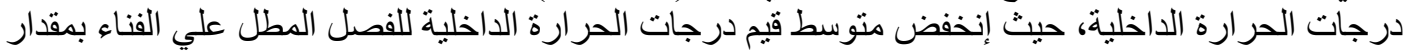
(0,6 كلفن) عن المكتب المطل علي الواجهة الخارجية في الدور الثناني.

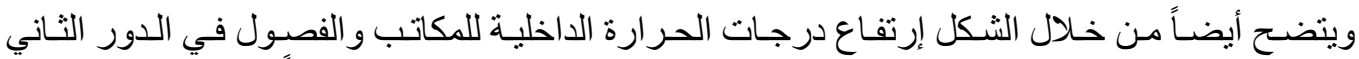

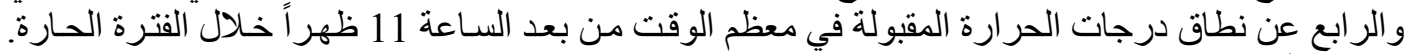

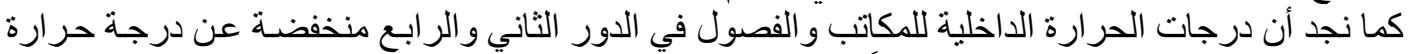

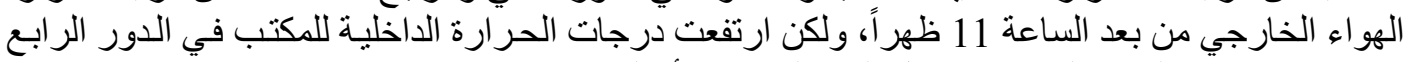
عن درجة حرارة الهو اء الخارجي خلال اليوم الدر اسي بأكمله. 

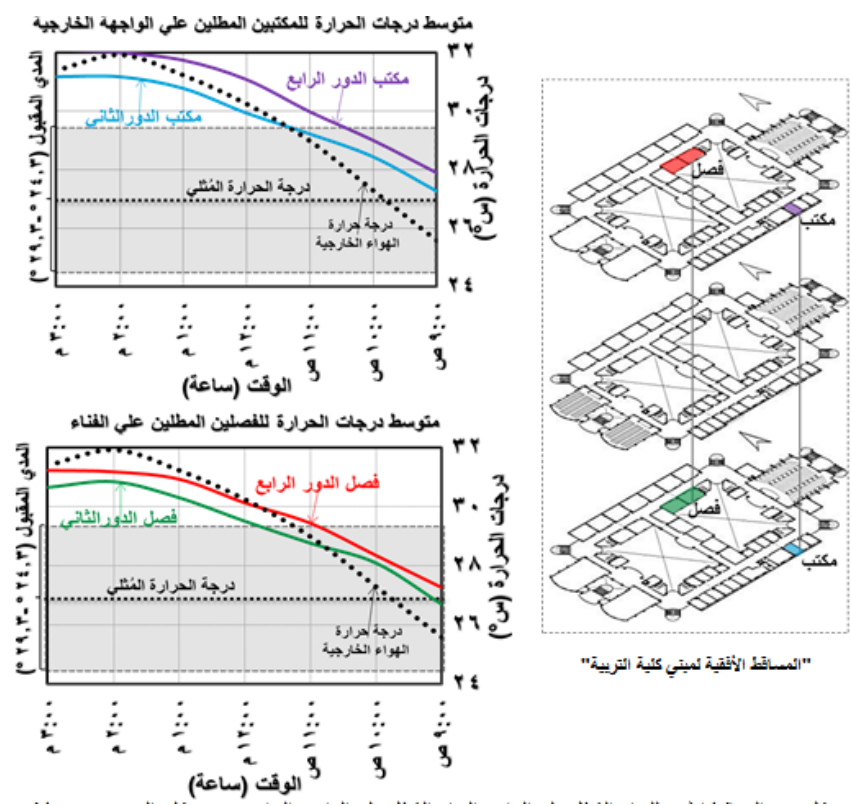

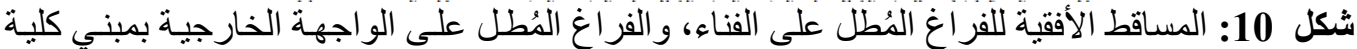

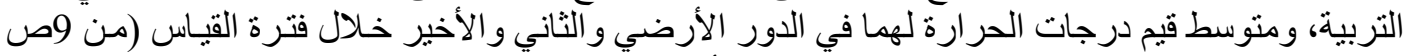

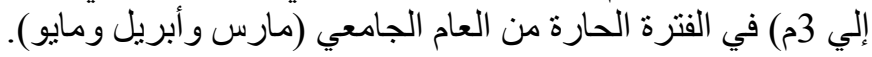

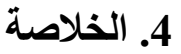

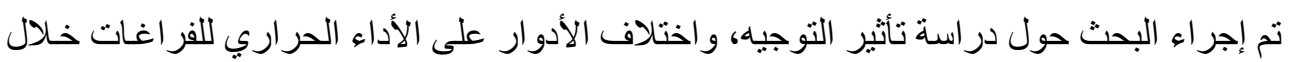

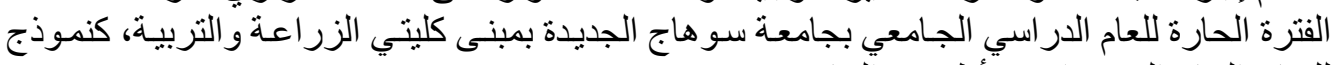

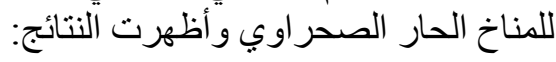

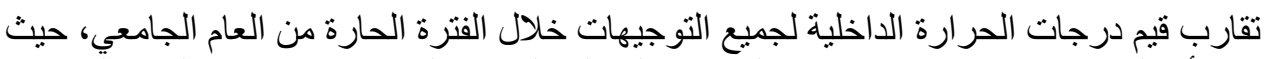

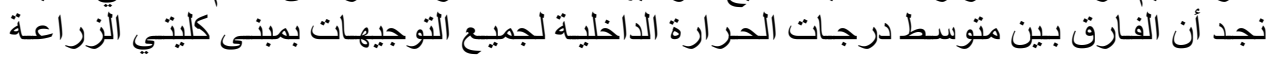

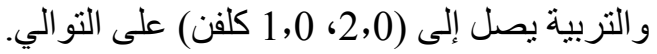

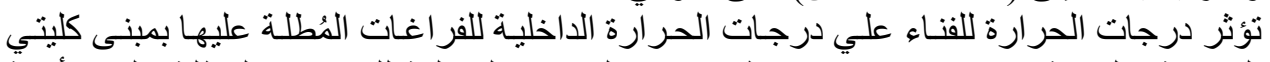

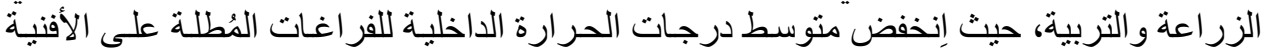

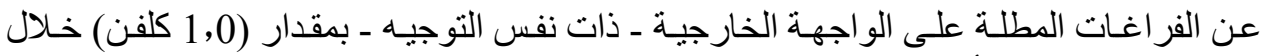

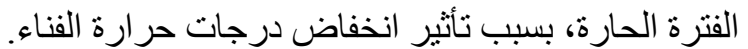

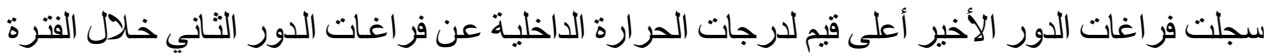

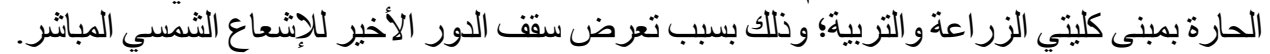

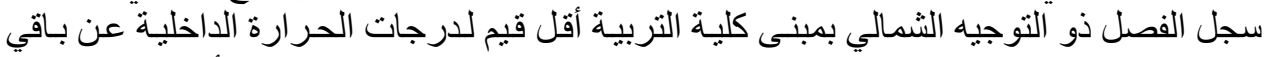

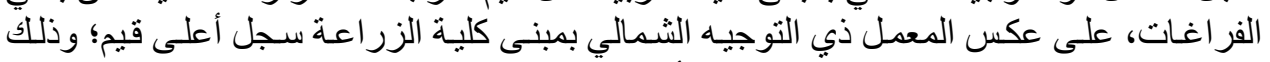

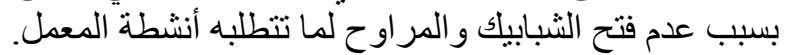

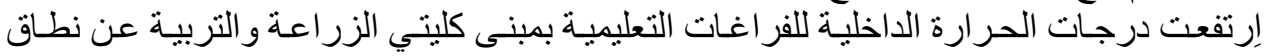

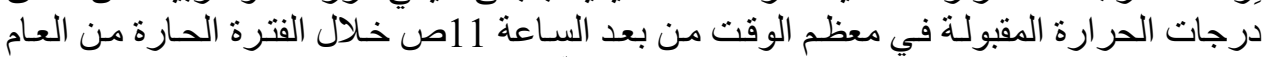

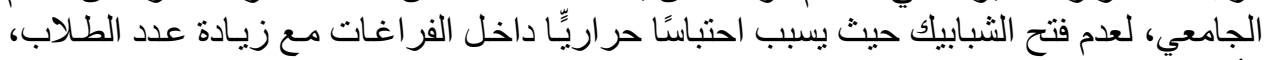

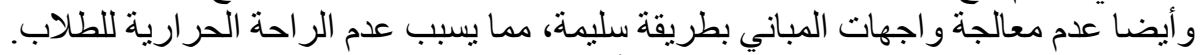

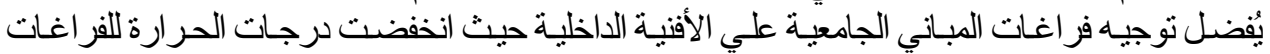

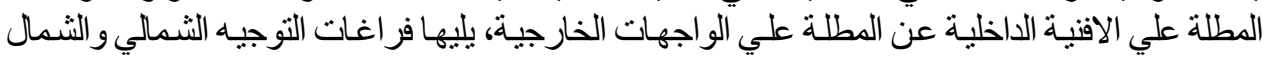




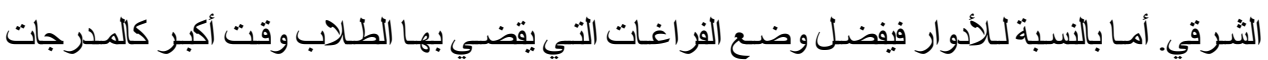
و القاعات الدر اسية والفصول في الأنوار المنخفضة كالدور الثاني، ووضع باتي الفي الفراغات في باقي الأدوار.

ـ توصيات خاصة بمبني كليتي الزر اعة و التربية في جامعة سو هاج الجديدة:

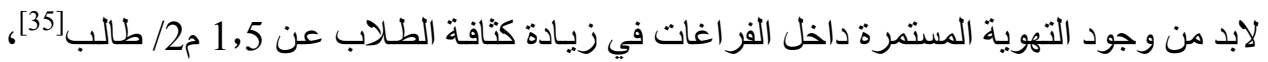

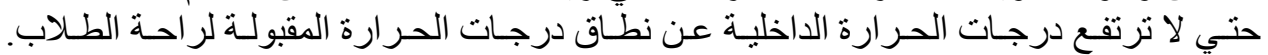

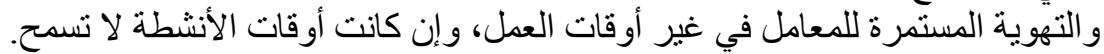

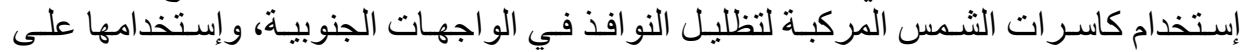

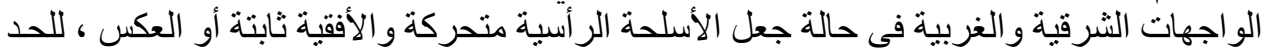

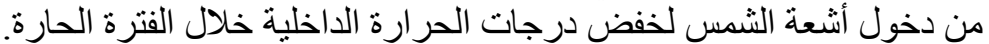

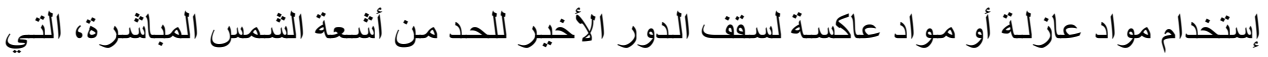
ثُؤثر علي الر احة الحرارية للطلاب.

ـ توصيات خاصة بتصميم المباني في المناطق الحارة الجافة لتحقيق الراحة الحرارية داخل فر اغات المباني الجامعية:

إستخدام الأفنية الداخلية في تصميم المباني الجامعية و الإهتمام بتنسيقها بزر اعتها ووضــع العناصـر

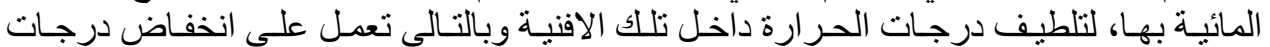

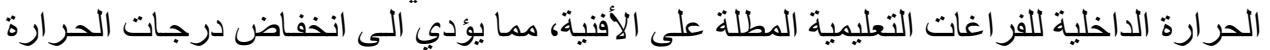
داخل الفر اغات المطلة علي تلك الأفنية.

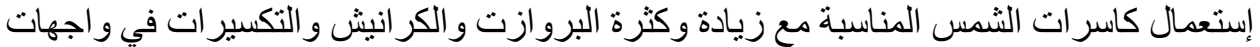

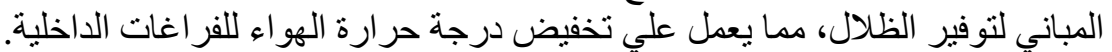

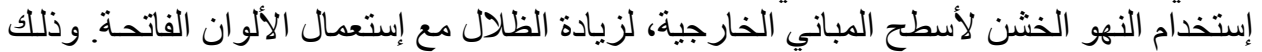

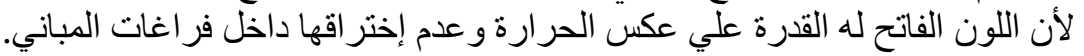

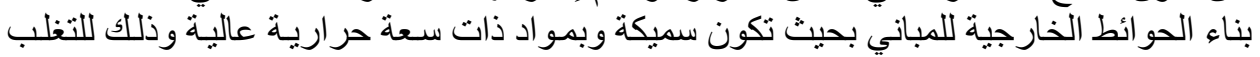

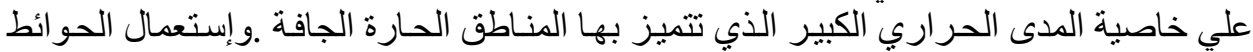

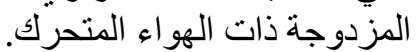

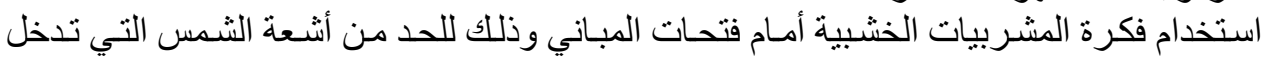

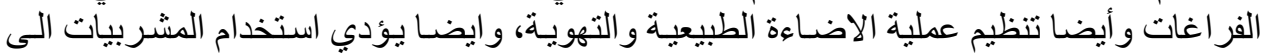
زيادة الاظلال على واجن أنهات المباني.

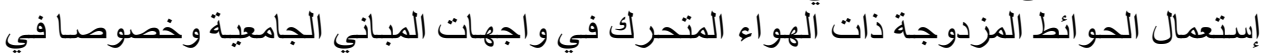

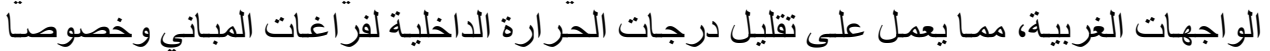
الفر اغات المطلة على الو اجهات الغربية.

\section{6. الدراسات المستقبلية}

در اسة تأثير الأحمال الحراريـة علي الراحة الحر اريـة للطلاب داخل الفر اغات التعليمية بالمباني الجامعية في الفترة الحارة الحمان.

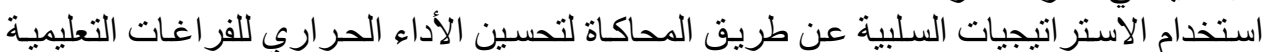

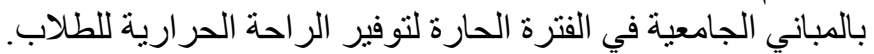

[1] Taleb. H, Sharples. S. (2011). Developing sustainable residential buildings in Saudi Arabia: A case study. Applied Energy, 88(1), 383-391. 


$$
\text { دينا أحدد محدد حسين وآخرون ـ تأثبير التوجيه واختلاف الأدوار على الأداء الحراري للفراغات الداخلبة. }
$$

[2] Budiaková, M. (2017). Evaluation of Indoor Climate in Small University Lecture Hall. Applied Mechanics and Materials, Trans Tech Publ.

[3] Amasuomo, T., Amasuomo, J. (2016). Perceived thermal discomfort and stress behaviours affecting students' learning in lecture theatres in the humid tropics. Buildings. 6(2), 18.

[4] Sullivan, P., \& Trujillo, A. (2015). The Importance of Thermal Comfort in the Classroom. United States Military Academy.

[5] Marchand, G., Nardi, M., and et al. (2014). The impact of the classroom built environment on student perceptions and learning. Journal of Environmental Psychology, 40, 187-197.

[6] Yong Thau Foong, S. (2008). Measurement Of Thermal Comfort At Classroom: A Case Study In UTeM. (Doctoral dissertation, UTeM).

[7] AMASUOMO, T., AMASUOMO, J. (2016). Thermal comfort problems in teaching-learning lecture theatres in the tropics: need to establish design criteria. wseas transactions on environment and development, 2(2), 12 .

[8] Samaan, M., Farag, o and Khalil, M. (2011). towards green campuses in egypt using simulation tools for optimization of drawing halls. AASHE ,Pittsburgh PA, USA.

[9] Abdallah, A. (2015). Analysis of Thermal Comfort and Energy Consumption in Long Time Large Educational Halls (Studios). Assiut University, Egypt, Procedia Engineering, 121, 16741681.

[10] Konya. A. (1980). Design Primer for Hot Climates. The Architectural Press Ltd, London.

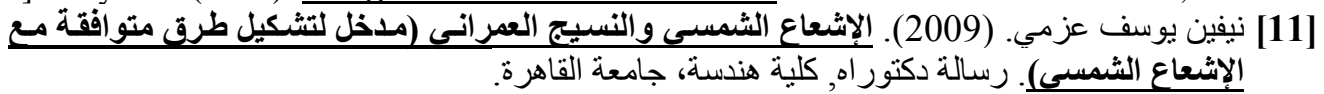

[12] Ali, A. (2012). Using simulation for studying the influence of vertical shading devices on the thermal performance of residential buildings (Case study: New Assiut City). Ain Shams Engineering Journal, 3(2), 163-174.

[13] Elgindi, S. (2010). The effect of building envelope design on energy conservation. Mc. s. thesis, Cairo University, Egypt.

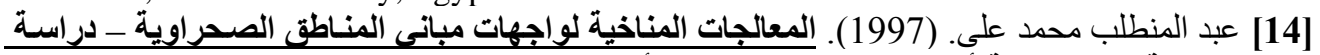

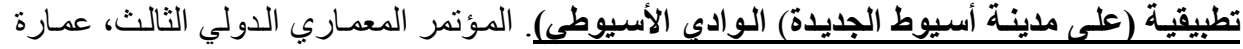

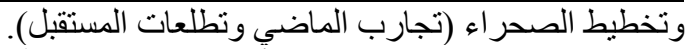

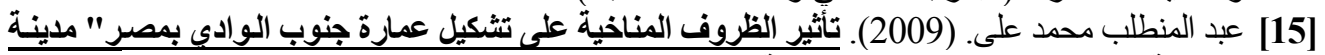

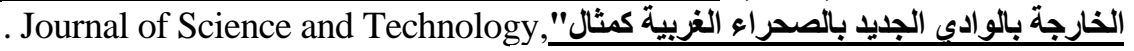

[16] Hamdani. M, Bekkouche. S, Benouaz. T\& Belarbi. R. (2014). Minimization of indoor temperatures and total solar insolation by optimizing the building orientation in hot climate. Engineering Structures and Technologies, 6(3), 131-149.

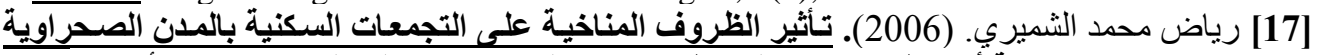

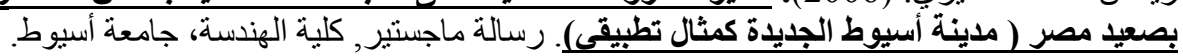

[18] Ponni. M, Baskar. R. (2014). Evaluation of Indoor Temperature through Roof and Wall Temperatures-An Experimental Study in Hot and Humid Climate. Evaluation, 4(6).

[19] Ponni. M, Baskar. R. (2015). A study of comfort temperature and thermal efficiency of buildings. The International Journal of Advanced Manufacturing Technology. 7, 1469-1477.

[20] ASHRAE: Cooling And Heating Load Calculations - Estimation Of Solar Radiation, ASHRAE Handbook-Fundamentals, American Society of Heating, Refrigerating and AirConditioning Engineers, Inc. Chapter 17, 2009.

[21].Ponni. M, Baskar. R. (2015). A Study on Indoor Temperature and Comfort Temperature. The International Journal of Advanced Manufacturing Technology. 4(3), 7-14. [22] أحمد عبد المنطلب. (2011). إستخدام المحاكاة لتقييم وتحسين الأداء الحراري للمبانى السكنية (دراسة

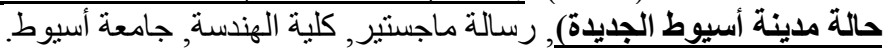

[23] Ponni. M, Baskar. R. Summer Thermal Performance of a Multistoried Residential Building. Eng. Sci. Invention, 6(2), 1-7.

[24] Farghal, A. A. F. (2011). Studying the Adaptive Comfort Model A Case Study in Arid Climate: Cairo, Egypt (Doctoral dissertation, Karlsruhe Institute of Technology). 
[25] Dewidar, K.M., Mahmoud, A.H., Moussa.(2013). Enhancing the human thermal comfort in-side educational buildings in hot arid regions, International conference of global climate change, biodiversity and sustainability, challenges and opportunities, AAST Egypt.

[26] Huang, K. T., Huang, W. P., Lin, T. P., \& Hwang, R. L. (2015). Implementation of green building specification credits for better thermal conditions in naturally ventilated school buildings. Building and Environment, 86, 141-150.

[27] Mishra, A. K., \& Ramgopal, M. (2015). A thermal comfort field study of naturally ventilated classrooms in Kharagpur, India. Building and Environment, 92, 396-406.

[28] Krawczyk, D. A., Gładyszewska-Fiedoruk, K., \& Rodero, A. (2017). The analysis of microclimate parameters in the classrooms located in different climate zones. Applied Thermal Engineering, 113, 1088-1096.

[29] HBRC. Code: ECP 306-2005, (2006). The Egyptian Code for enhancing energy use in buildings, Housing and Building Research Center (HBRC), Cairo, Egypt.

[30] https://www.climate-charts.com/Locations/e/UB62397, (cited January 2020).

[31] http://www.noaa.gov/ , (cited June 2018).

[32] https://www.meteoblue.com, (cited June 2019).

[33] Smith. E, Daivis. P \& Todd. B. (2019). Measuring heat flux beyond Fourier's law. The Journal of chemical physics, 150(6), 064103.

[34] https://www.omnicalculator.com/physics/thermal-conductivity\#fouriers-law-and-heat-flux, (cited June 2019).

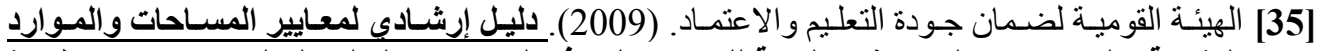

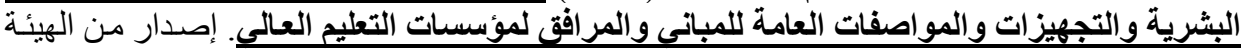

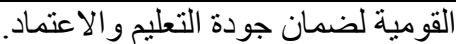

\title{
THE EFFECT OF ORIENTATION AND ORDERING FLOORS ON THE THERMAL PERFORMANCE OF INTERNAL SPACES IN THE FACULTIES OF AGRICULTURE AND EDUCATION AT NEW SOHAG UNIVERSITY DURING THE HOT PERIOD
}

\begin{abstract}
The research aims to study the effect of orientation and different floors on the thermal performance of spaces in university buildings during the hot period of the university year in the building of the Faculties of Agriculture and Education at New Sohag University. To achieve the objective of the study, the research depends on the analytical method and field measurements, which includes field measurements, so (outdoor and indoor temperatures, and relative humidity) ) were measured for the spaces of the Faculties of Agriculture and Education overlooking the yards as well as on the external facades when the different orientations and floors, which represents the measurement of 6 days of each month (March, April, May). The results showed the convergence of indoor temperatures for all directions during the hot period in the building of the Faculties of Agriculture and Education, where we find that the average difference is not more than $2 \mathrm{k}^{\circ}$ because the ventilation is insufficient in the spaces. The indoor temperatures of the spaces overlooking the courtyards of the Faculty of Agriculture and Education on the outdoor were reduced by an average of $1.02 \mathrm{k}^{\circ}$ during the hot period. The spaces of the final floor also recorded higher of indoor temperature than the second floor spaces during the hot period in the faculty of agriculture and education building, in order to expose the ceiling of the final floor of direct solar radiation. The indoor temperatures of the spaces with different directives in the Faculties of Agriculture and Education building during the hot period are also shown to be higher than the range of temperatures accepted most of the time after 12:00 pm (which represents $50 \%$ to $60 \%$ ). Of the total number of hours of measurement during the hot period of the academic year. Therefore, it's recommended that there is continuous ventilation in the educational spaces so that indoor temperatures don't rise above the range of indoor temperatures acceptable for the comfort of students, which affects their performance and educational achievement.
\end{abstract}

Keywords: Thermal comfort - university buildings - thermal performance - orientation - different floors. 